MATHEMATICS OF COMPUTATION

Volume 72 , Number 241 , Pages 159-181

S 0025-5718(02)01438-2

Article electronically published on August 13, 2002

\title{
A LEVEL SET APPROACH FOR COMPUTING DISCONTINUOUS SOLUTIONS OF HAMILTON-JACOBI EQUATIONS
}

\author{
YEN-HSI RICHARD TSAI, YOSHIKAZU GIGA, AND STANLEY OSHER
}

\begin{abstract}
We introduce two types of finite difference methods to compute the L-solution and the proper viscosity solution recently proposed by the second author for semi-discontinuous solutions to a class of Hamilton-Jacobi equations. By regarding the graph of the solution as the zero level curve of a continuous function in one dimension higher, we can treat the corresponding level set equation using the viscosity theory introduced by Crandall and Lions. However, we need to pay special attention both analytically and numerically to prevent the zero level curve from overturning so that it can be interpreted as the graph of a function. We demonstrate our Lax-Friedrichs type numerical methods for computing the L-solution using its original level set formulation. In addition, we couple our numerical methods with a singular diffusive term which is essential to computing solutions to a more general class of HJ equations that includes conservation laws. With this singular viscosity, our numerical methods do not require the divergence structure of equations and do apply to more general equations developing shocks other than conservation laws. These numerical methods are generalized to higher order accuracy using weighted ENO local Lax-Friedrichs methods as developed recently by Jiang and Peng. We verify that our numerical solutions approximate the proper viscosity solutions obtained by the second author in a recent Hokkaido University preprint. Finally, since the solution of scalar conservation law equations can be constructed using existing numerical techniques, we use it to verify that our numerical solution approximates the entropy solution.
\end{abstract}

\section{Introduction}

Nonlinear Hamilton-Jacobi equations arise in many different fields, including mechanics, calculus of variations, geometric optics, control theory, and differential games. Because of the nonlinearity, the Cauchy problems usually have nonclassical solutions due to the crossing of characteristic curves.

For scalar equations of conservation law type, there is a well known theory regarding the existence and uniqueness of a weak solution, called an entropy solution, using the special integral structure of the equation [23]. Advanced numerical methods, e.g., [15], [16], [30], [34], have been developed and widely used to compute approximations that converge to the correct entropy solutions.

Received by the editor March 7, 2001.

2000 Mathematics Subject Classification. Primary 65Mxx, 35Lxx; Secondary 70H20.

Key words and phrases. Hamilton-Jacobi equations, singular diffusion, level sets.

The first and the third authors are supported by ONR N00014-97-1-0027, DARPA/NSF VIP grant NSF DMS 9615854 and ARO DAAG 55-98-1-0323. 
Nevertheless, this notion of weak solution cannot be applied to many fully nonlinear equations, e.g., the eikonal equation $u_{t}+|\nabla u|=0$. In 1983, Crandall and Lions [7] first introduced the notion of viscosity solution for this type of equations, based on a maximum principle and the order-preserving property of parabolic equations. In general, for any given Hamilton-Jacobi equation of the form

$$
u_{t}+H(x, t, u, D u)=0,
$$

where $H$ is a continuous function from $\Omega \times \mathbb{R}^{+} \times \mathbb{R} \times \mathbb{R}^{n}$, nondecreasing in $u$, and $\Omega$ is an open subset of $\mathbb{R}^{n}$, there exists a unique uniformly continuous viscosity solution if the initial data is bounded and uniformly continuous 1 The continuity of the solution can be understood intuitively from the 1D fact that "HJ equations are the conservation laws integrated once." The viscosity solution is sometimes understood as the limit of the solutions to the equation with vanishing viscosity.

Correspondingly, Crandall and Lions in [6] proved the convergence of two approximations to the viscosity solution of equations whose Hamiltonians only depend on $D u$. This was generalized by Souganidis to equations with variable coefficients in 31. Many sophisticated numerical methods have since been developed [21, 24], [26], 27].

However, there are problems in control theory and differential games which demand discontinuous solutions. The original viscosity theory does not apply to discontinuous initial data. The notion of semicontinuous viscosity solution has been introduced first by Ishii [18, 20] using an extension of Perron's method. Because of the nonuniqueness in Ishii's result, other notions of semicontinuous solutions were proposed by various authors [2], 4], with different kinds of additional properties imposed on the Hamiltonian. Some of these notions need serious restrictions on the Hamiltonians, and others are implicit in the sense that the processes of taking supremum and infimum are involved. As a consequence, one cannot develop numerical methods to construct approximations. For an overview of the viscosity theory and applications, see 3 and $[1$.

Finally, for the class of equations with Hamiltonians $H(x, u, D u)$ nondecreasing in $u$, M.-H. Sato and the second author [14] introduced a new notion of semicontinuous solution. This notion of solution is defined by the evolution of the zero level curve of the auxiliary level set equation which embeds the original HJ equation. It is thus called the L-solution. In this article, we will devise a Lax-Friedrichs type scheme to compute approximation of the L-solution in its original formulation (i.e., level set). We will also show that with suitable CFL condition, our schemes keep the discrete version of an important property of this class of HJ equations.

When the Hamiltonian $H(t, x, u, D u)$ is not nondecreasing in $u$, the solution may develop shocks in finite time even if the initial data is continuous. Recently, a new notion called the proper viscosity solution was introduced by the second author 13 to track the whole evolution. This notion is consistent with the entropy solution when the equation is a conservation law. In order to approximate the proper viscosity solution of a class of more general HJ equations, we introduce a singular diffusive term in the vertical direction to the auxiliary level set equations so that the level curves will not overturn. In the case of conservation laws, the proper viscosity solution is consistent with the entropy solution. We will show numerically that the shock solutions we obtain from the regularized level set equations satisfy the "equal

\footnotetext{
${ }^{1}$ Notice that the conservation laws do not fall into this category because the corresponding $H$ might not be monotone in $u$; e.g., shocks may develop from smooth initial data.
} 
area" entropy condition, and thus demonstrate the validity of our regularization terms. We emphasize that, based on our numerical results, the global property of our singular diffusion term regularizes our nonconservative level set equations so that the entropy condition is satisfied during the time iterations.

We remark that a simple monotone Lax-Friedrichs scheme seems to produce convergent approximations of the L-solution for the first class of HJ equations in their original form, even though the scheme does not follow the original definition of the L-solution. However, for the second class of equations, it is likely that the numerical approximations obtained this way converge to the wrong weak solution. This is a well known fact for monotone schemes for conservation laws in nonconservative form. In contrast, our numerical approximations for the corresponding "nonconservative" level set equations appear to converge to the right weak solution; i.e., the proper viscosity solution and, in case of conservation laws, the entropy solution.

In the following sections, we first review briefly the previous work on using level sets as a tool to analyze and compute solutions of given PDEs. We then derive the level set equation from a given HJ equation. We then devise numerical methods for the level set equations for the computation of the solutions of the HJ equations according to the behavior of $H_{u}$. We extend each type of our numerical schemes to higher order accuracy using the WENO schemes devised in [21].

1.1. Analysis by the level set function. Osher 25] rediscovered a method of Jacobi [5] to study the Cauchy problem for general first order nonlinear equations through the aid of the level set equations. In that paper, Osher derived from the general first order equation

$$
F\left(x, y, u, u_{x}, u_{y}\right)=0
$$

a time-dependent Hamilton-Jacobi equation

$$
\phi_{t}+H\left(x, y, t, \phi_{x}, \phi_{y}\right)=0
$$

and proved that the zero level set of its solution at time $t$ is the set $\{(x, y): u(x, y)=$ $t\}$. With continuous initial values, the viscosity solution theory gives the existence and uniqueness of the solution to the time-dependent Hamilton-Jacobi equations provided that $H$ does not change sign.

In [8], Evans used the level set method described in [25] to obtain the level surface heat equation. He gave the geometric interpretation of the instant "unfolding" of multi-valued initial data of the solution of the linear heat equation. By considering the viscous Burgers' equation

$$
u_{t}+u u_{x}=\varepsilon u_{x x}, \quad \varepsilon>0,
$$

as a lower order perturbation to the heat equation, Evans provided further analysis and a geometrical explanation as to how the term $\varepsilon u_{x x}$ keeps the solution from becoming multi-valued.

Recently, M.-H. Sato and the second author proposed to characterize the semicontinuous solutions of HJ equations using a similar approach. In their paper [14, they define the L-solution and prove its existence and uniqueness with a class of Hamiltonians. We remark that the L-solution is equivalent to the conventional viscosity solution if the hypotheses are identical.

The idea is to represent the "graph" of a semicontinuous function $u(x)$ as the zero level set of a function $\phi: \mathbb{R}^{2} \rightarrow \mathbb{R}^{1}$ with the requirement that every level set of $\phi$ is the graph of some function of $x$. More precisely, we define the subgraph 
of a function $u$ be be $\operatorname{sg}(u):=\left\{(x, y) \in \mathbb{R}^{2}: y \leq u(x)\right\}$ and the curve $\Gamma(t)$ to be the upper boundary of $\operatorname{sg}(u)$. For smooth functions $u(x, t), \Gamma(t)$ is simply the graph of $u$ at time $t$. The numerical construction of such functions is described in the appendix.

Consider the general first order equation

$$
u_{t}+H\left(t, x, u, u_{x}\right)=0,
$$

where $u$ is a function from $\mathbb{R} \rightarrow \mathbb{R}$. Embed $\Gamma(t)$ as the zero level set of a function $\phi: \mathbb{R}^{2} \times \mathbb{R}^{+} \rightarrow \mathbb{R}$; i.e., $\phi(t, x, y)=0$ for all $(x, y) \in \Gamma(t)$ for each $t \in \mathbb{R}^{+}$. Taking partial derivatives, we have

$$
\begin{aligned}
& 0=\frac{d}{d t} \phi(t, x, y)=\frac{d}{d t} \phi(t, x, u(t, x))=\phi_{t}+\phi_{y} \cdot u_{t} ; \\
& 0=\frac{d}{d x} \phi(t, x, y)=\frac{d}{d x} \phi(x, u(t, x))=\phi_{x}+\phi_{y} \cdot u_{x} .
\end{aligned}
$$

Therefore, we have formally $u_{t}=-\phi_{t} / \phi_{y}$ and $u_{x}=-\phi_{x} / \phi_{y}$, and equation (1.1) becomes

$$
\phi_{t}-\phi_{y} H\left(t, x, y,-\frac{\phi_{x}}{\phi_{y}}\right)=0
$$

Under the hypotheses described in the next section, equation (1.2), together with a Lipschitz continuous initial function $\phi_{0}$ which embeds the initial data $u_{0}$, is in the class of HJ equations that is known to have continuous solution $\phi(x, y, t)$.

However, in order to interpret the zero level set $\Gamma(t)$ of $\phi(x, y, t)$ as the evolution of the "graph" of $u(x, t), \Gamma(t)$ has to be a one-to-one mapping of the variable $x$. We will call this requirement "nonoverturning". If $\phi(x, y, 0)$ is set up with $\phi_{y}(x, y, 0) \geq 0$ everywhere, the nonoverturning requirement is equivalent to the condition $\phi_{y}(x, y, t) \geq 0$ for all $t \geq 0$. In devising numerical approximations, it is important to make sure that this condition is true discretely.

In the following sections, we will use $H_{u}$ to denote the partial derivative of $H\left(x, u, u_{x}\right)$ with respect to $u$ for the original HJ equations. Finally, the level set function $\phi$ is set up to be nondecreasing in $y$ initially in the examples of this paper.

\section{Model Equations}

We first consider the scalar 1D equation

$$
u_{t}+H\left(x, u, u_{x}\right)=0
$$

with the Hamiltonian $H\left(x, u, u_{x}\right)$ satisfying the following properties:

1. $H$ is Lipschitz in all its arguments.

2. $\lim _{\lambda \rightarrow 0} \lambda H(x, u, p / \lambda)$ exists.

In addition, we are concerned with the following two classes of equations: 1) equations with $H_{u} \geq 0$ but with discontinuous initial data; 2) equations such as conservation laws that do not belong in the first class.

Let us consider the following two model equations, both of which can be in either the first or the second class depending on the parameters:

- Equations that contains both terms from conservation laws and fully nonlinear first order terms:

$$
u_{t}+u u_{x}+a u\left|u_{x}\right|=0, a \in \mathbb{R}
$$


The associated level set equation is

$$
\phi_{t}-y \cdot\left(a \operatorname{sign}\left(\phi_{y}\right)\left|\phi_{x}\right|-\phi_{x}\right)=0 .
$$

We can see that the value of $a$ determines an important property of $H_{u}$ : for $a \geq 1, H_{u}$ will be nondecreasing. Thus the viscosity theory applies if the initial data is uniformly continuous, and we know the solution will be uniformly continuous. This falls into the first class of equations. For $0 \leq|a|<1, H_{u}$ changes signs according to the value of $u_{x}$. Then equation (2.1) belong to the second class. Notice that if $a=0$, we have the inviscid Burgers' equation.

- Equations that prescribe the normal motion of the graph of $u$ :

$$
u_{t}-v(u) \sqrt{1+u_{x}^{2}}=0 .
$$

The corresponding level set equation is

$$
\phi_{t}+\operatorname{sign}\left(\phi_{y}\right) v(y)|\nabla \phi|=0
$$

The function $v$ is the normal velocity of the graph of $u$, or the level sets of $\phi$. If $v$ ever decreases, then $H_{u} \leq 0$ and the equation fails to be in the first class.

The role of $\operatorname{sign}\left(\phi_{y}\right)$. Let us look at the characteristics of equation (2.4) more carefully. The term $\operatorname{sign}\left(\phi_{y}\right)$ flips the direction of the characteristics whenever $\phi_{y}$ changes signs. If the characteristics on the upper part of the jump travel faster than those on the lower part (i.e., $v(y)$ is increasing), the overturning will develop. With the $\operatorname{sign}\left(\phi_{y}\right)$ term, whenever overturning just happens, the direction of a characteristic will be reversed, making it travel backward and thus eliminate the overturning. However, this fact is not directly suitable for numerical implementation.

2.1. Geometrical explanation of the nonoverturning conditions. As mentioned earlier, we need to pay special attention in order to prevent the overturning of the level curves of $\phi$. One equivalent criterion is to demand the minimum principle: $\phi_{y}(x, y, t) \geq 0$ for $t \geq 0$.

In light of the level set equation (2.4), we have a more geometrical requirement on the speed function $v$. By the method of characteristics, we know that $v(y)$ prescribes the normal velocity of the level sets of $\phi$. On the vertical segments of the level sets, which correspond to jumps in $u, v(y)$ prescribes the horizontal velocity according to $y$. Overturning will happen if $v(y)$ is increasing, since the upper part of the jump of $u$ moves faster than the lower part. See Figure1.

Consider the primitive function of $v$ :

$$
V(y)=\int v(s) d s .
$$

The nonincreasing condition of $v$ translates to the concavity of $V$ ! This fact reminds us of one of the entropy conditions for conservation laws with nonconcave flux function. It says that the entropy solution of a conservation law with nonconvex flux $f$ is the classical solution of the conservation law with the flux $f^{*}$, where $f^{*}$ is the minimal concavification of $f$ over the increasing jump interval. This, in turn, provides a hint on the regularization of HJ equations (2.4) - we need to impose a regularization that concavifies the primitive function on the vertical segments of the level sets and nowhere else. We shall demonstrate numerically that our proposed singular diffusive regularization term does exactly that in a later part of this article. 


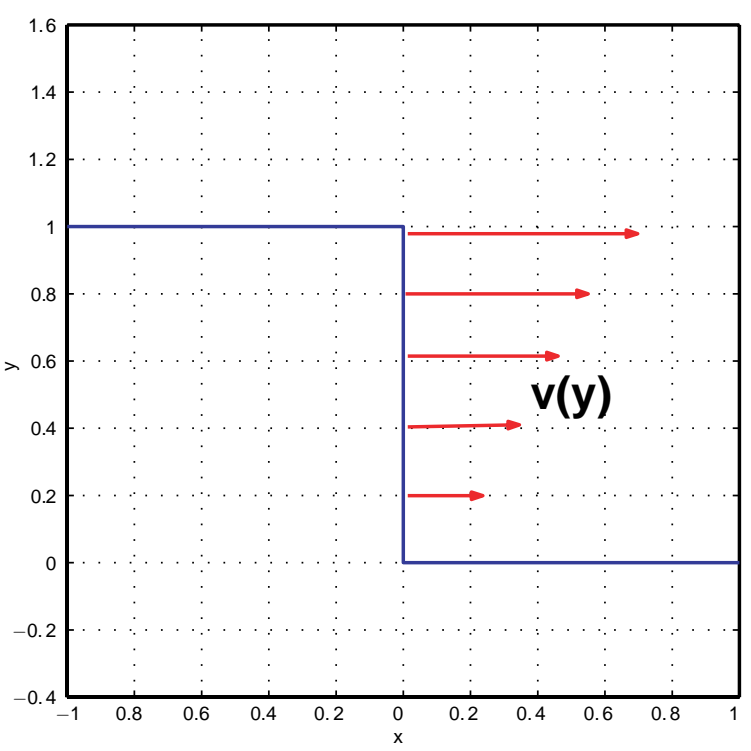

FIGURE 1. Overturning is caused by the normal velocity, which is increasing in the $y$-direction.

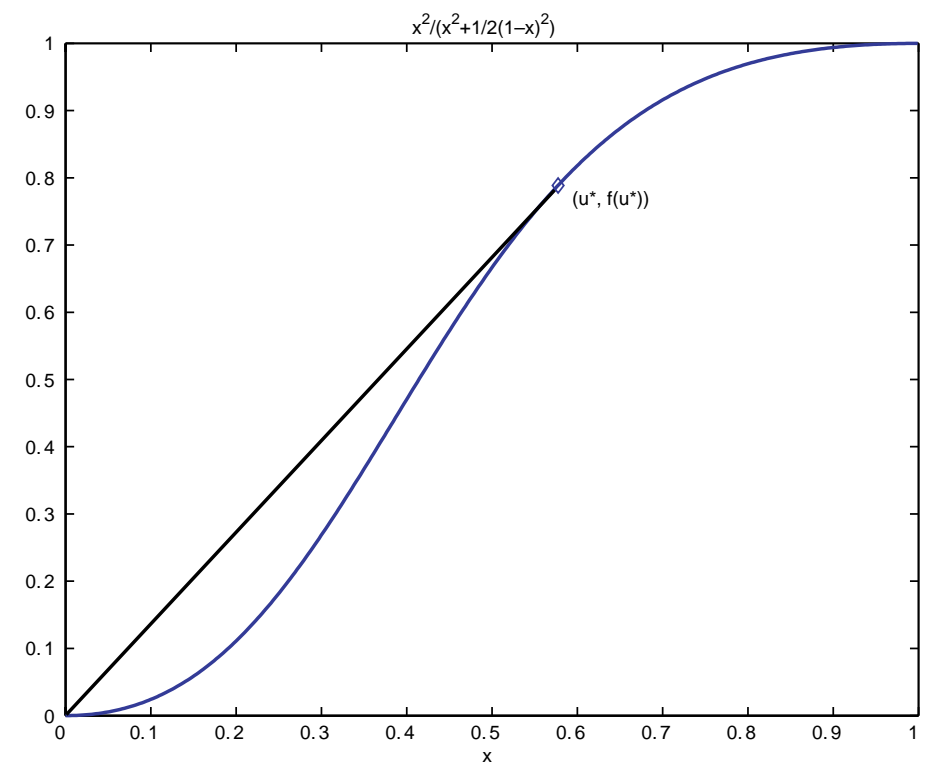

FiguRE 2. The concavification of the flux in the Buckley-Leverett equation.

2.2. Equations with Hamiltonian $H_{u} \geq 0$. We first consider the equations for which $H_{u} \geq 0$, and the corresponding level set equation. Equation (1.2) can be simplified to

$$
\phi_{t}-\tilde{H}\left(t, x, y, \phi_{x}, \phi_{y}\right)=0 .
$$


For example, with the homogeneity hypothesis, the factor $\phi_{y}$ in (1.2) can be brought into the original Hamiltonian $H\left(x, y, \phi_{x} / \phi_{y}\right)$, which then transforms into a new Hamiltonian $\tilde{H}\left(x, y, \phi_{x}, \phi_{y}\right)$. The reduction from equation (2.3) to equation (2.4) is one such example.

The minimum principle. The assumption that the Hamiltonian is nondecreasing in $u$ has an important consequence. We present here an argument about this minimum principle based on an argument in 14. Consider $\phi^{h}(x, y, t):=\phi(x, y+h, t)$, where $h>0$ and $\phi(x, y, t)$ is the uniformly continuous viscosity solution of equation (2.5) with uniformly continuous initial data $\phi_{0}(x, y)$. By definition, $\phi^{h}$ is the viscosity solution of

$$
\phi_{t}-\tilde{H}\left(t, x, y+h, \phi_{x}, \phi_{y}\right)=0
$$

with initial data $\phi_{0}^{h}(x, y)$. Let $v$ be a $C^{1}$ function; then at any local minimum of $\phi^{h}-v$

$$
v_{t}-\tilde{H}\left(t, x, y+h, v_{x}, v_{y}\right) \geq 0 .
$$

It is clear that if $H_{y} \geq 0$, then $\tilde{H}_{y} \geq 0$, where $\tilde{H}$ is the Hamiltonian of the corresponding level set equation. Consequently, we have

$$
v_{t}-\tilde{H}\left(t, x, y, v_{x}, v_{y}\right) \geq v_{t}-\tilde{H}\left(t, x, y+h, v_{x}, v_{y}\right) \geq 0
$$

at any local minimum of $\phi^{h}-v$ for any $C^{1}$ test function $v$. Thus $\phi^{h}$ is a viscosity supersolution of equation 2.5.

If $\phi^{h}(x, y, 0)-\phi(x, y, 0) \geq 0$ for all $x$ and $y$, then $\phi^{h}(x, t) \geq \phi(x, t) \geq 0$ by the comparison principle (the reader is referred to [11] and [19] for the proof). This basically says that if $\phi_{y}(x, y, t=0) \geq 0$ initially, then $\phi_{y}(x, y, t) \geq 0$ for all time! It also implies that $\{\phi=c\}$ will remain as a graph throughout the evolution. Therefore, we can remove the $\operatorname{sign}\left(\phi_{y}\right)$ term from the derived level set equation (1.2) of this class of equations.

Without causing confusion, we shall continue using the notation $H\left(x, y, \phi_{x}, \phi_{y}\right)$ in place of $\tilde{H}\left(x, y, \phi_{x}, \phi_{y}\right)$ in the following parts of this article.

The Lax-Friedrichs schemes for the level set equation. Following the methods originally conceived for HJ equations $\phi_{t}+H(D \phi)=0$ in [27], see also [26], and suppressing the dependence of $H$ on $x$ and $y$, we use the Local Lax-Friedrichs (LLF) flux

$$
\begin{aligned}
\hat{H}^{L L F}\left(p^{+}, p^{-}, q^{+}, q^{-}\right)= & H\left(\frac{p^{+}+p^{-}}{2}, \frac{q^{+}+q^{-}}{2}\right) \\
& -\frac{1}{2} \alpha^{x}\left(p^{+}, p^{-}\right)\left(p^{+}-p^{-}\right)-\frac{1}{2} \alpha^{y}\left(q^{+}, q^{-}\right)\left(q^{+}-q^{-}\right),
\end{aligned}
$$

for the approximation of $H$. In the above scheme,

$$
\begin{gathered}
\alpha^{x}\left(p^{+}, p^{-}\right)=\underset{p \in I\left(\left(p^{+}, p^{-}\right), C \leq q \leq D\right.}{\max }\left|H_{\phi_{x}}(p, q)\right|, \\
\alpha^{y}\left(q^{+}, q^{-}\right)=\max _{q \in I\left(\left(q^{+}, q^{-}\right), A \leq p \leq B\right.}\left|H_{\phi_{y}}(p, q)\right|, \\
I(a, b)=[\min (a, b), \max (a, b)],
\end{gathered}
$$

and $p^{ \pm}, q^{ \pm}$are the forward and backward approximations of $\phi_{x}$ and $\phi_{y}$, respectively. 
We can use a simple forward Euler time discretization and obtain the fully discretized scheme

$$
\phi_{i, j}^{n+1}=\phi_{i}^{n}+\Delta t \hat{H}^{L L F}\left(x_{i}, y_{j}, D_{x}^{+} \phi_{i, j}^{n}, D_{x}^{-} \phi_{i, j}^{n}\right)
$$

for the level set equations with $H$ independent of $\phi_{y}$ (after removing $\operatorname{sign}\left(\phi_{y}\right)$ ), and

$$
\phi_{i, j}^{n+1}=\phi_{i}^{n}+\Delta t \hat{H}^{L L F}\left(x_{i}, y_{j}, D_{x}^{+} \phi_{i, j}^{n}, D_{x}^{-} \phi_{i, j}^{n}, D_{y}^{+} \phi_{i, j}^{n}, D_{y}^{-} \phi_{i, j}^{n}\right)
$$

for equations such as equation (2.3), since the Hamiltonians depend on $\phi_{y}$. Here, $\phi_{i, j}^{n}:=\phi\left(x_{i}, y_{j}, t_{n}\right)$, and $\Delta x, \Delta y$, and $\Delta t$ are the step size in $x, y$ and $t$.

Rewrite the above schemes in the form

$$
\phi_{i, j}^{n+1}=G\left(x_{i}, y_{j}, \phi_{i+1, j+1}^{n}, \phi_{i+1, j}^{n}, \phi_{i, j+1}^{n}, \phi_{i, j}^{n}, \phi_{i-1, j}^{n}, \phi_{i, j-1}^{n}, \phi_{i-1, j-1}^{n}\right) .
$$

If $G$ is nondecreasing in all its arguments except $x_{i}$ and $\Delta_{y}^{-} \phi_{i, j}^{n} \geq 0$ for all $i, j \in Z_{d}$, then

$$
\begin{aligned}
\Delta_{y}^{-} \phi_{i, j}^{n+1}= & \phi_{i, j}^{n+1}-\phi_{i, j-1}^{n+1} \\
= & G\left(x_{i}, y_{j}, \phi_{i+1, j+1}^{n}, \phi_{i+1, j}^{n}, \phi_{i, j+1}^{n}, \phi_{i, j}^{n}, \phi_{i-1, j}^{n}, \phi_{i, j-1}^{n}, \phi_{i-1, j-1}^{n}\right) \\
& \quad-G\left(x_{i}, y_{j-1}, \phi_{i+1, j}^{n}, \phi_{i+1, j-1}^{n}, \phi_{i, j}^{n}, \phi_{i, j-1}^{n}, \phi_{i-1, j-1}^{n}, \phi_{i, j-2}^{n}, \phi_{i-1, j-2}^{n}\right) \\
\geq & 0 .
\end{aligned}
$$

Because of the hypothesis that $H_{y} \geq 0$, our Lax-Friedrichs schemes preserve the minimum principle discretely (i.e., given $\Delta_{y}^{+} \phi_{i, j}^{n} \geq 0$ for all $i, j \in Z_{d}$, then $\Delta_{y}^{+} \phi_{i, j}^{n+1}$ $\geq 0$ for all $i, j \in Z_{d}$ ) if

$$
\frac{\Delta t}{\Delta x} \leq C \min \left(1 /\left\|H_{\phi_{x}}\right\|_{\infty}, 1 /\left\|H_{\phi_{y}}\right\|_{\infty}\right)
$$

where $C=1$ for equation (2.6) and $C=2$ for equation (2.7).

Extension to higher order of accuracy. To achieve higher order accuracy and have less numerical disspation, we can discretize the spatial derivatives using WENO schemes described in [21], which essentially replace the forward/backward differencing by higher order WENO approximations. For higher order accuracy in time discretization, the TVD third order Runge-Kutta method from [30] can be used.

2.2.1. Examples. We provide here some numerical computations for some equations that belong to the class we are considering.

Constant motion along the normal. Consider the equation

$$
u_{t}+c \sqrt{1+u_{x}^{2}}=0 .
$$

Given a continuous initial data, it is well-known that the following equation corresponds to motion of the graph with constant normal velocity $c$.

Using the notion of the L-solution, we can easily describe the motion defined by equation (2.8), even with piecewise continuous data. The corresponding level set equation is simply

$$
\phi_{t}+c|\nabla \phi|=0
$$

which describes the constant normal speed motion of each level set of $\phi$. We emphasize here that since the level sets of $\phi$ are continuous, we can simply use the existing classical viscosity theory for the solution. Figure 3 shows the zero level curves of $\phi$ in different times. The reader can see that each curve is equidistant from the original curve (shown as red). 


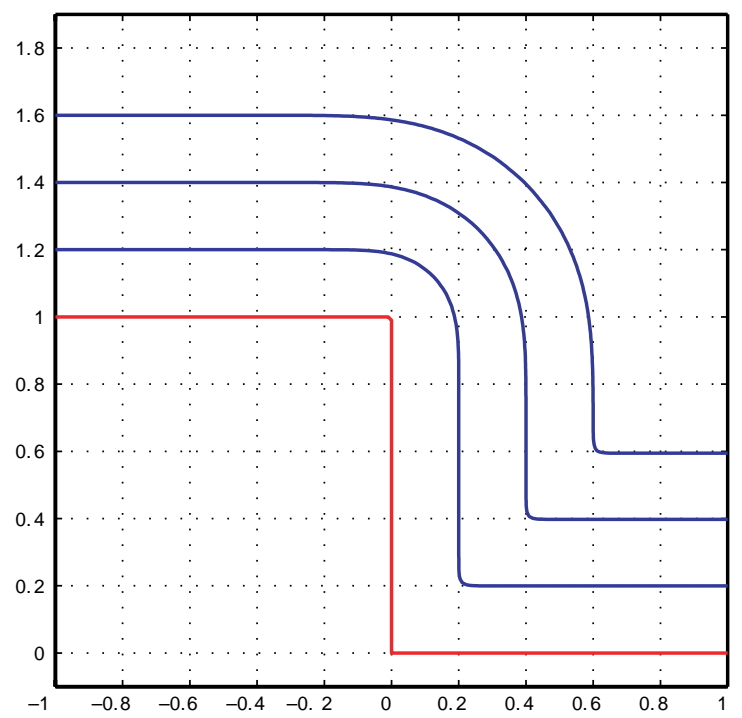

Figure 3. Numerical solution by first order LLF method for the Riemann problem for equation (2.8) with $u_{L}=1.0, u_{R}=0.0$, and $c=1.0$. We plotted the zero level set at times $t=0,0.2,0.4$ and 0.6 .

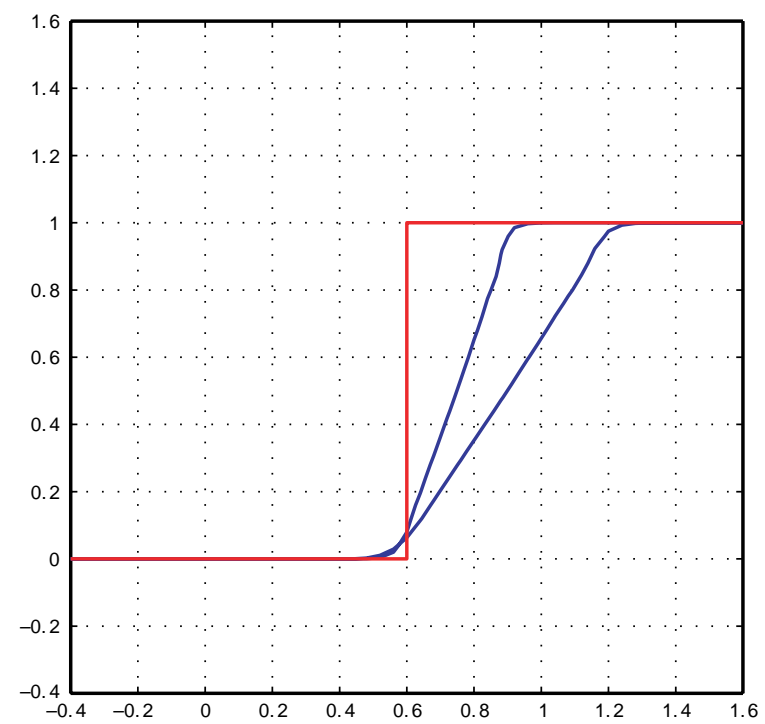

Figure 4. Numerical solution using third order WENO-LLF to the Riemann problem for equation (2.1) with $u_{L}=0.0, u_{R}=0.1$, and $a=2.0$. We plotted the zero level set at times $t=0,0.1$, and 0.2 . 


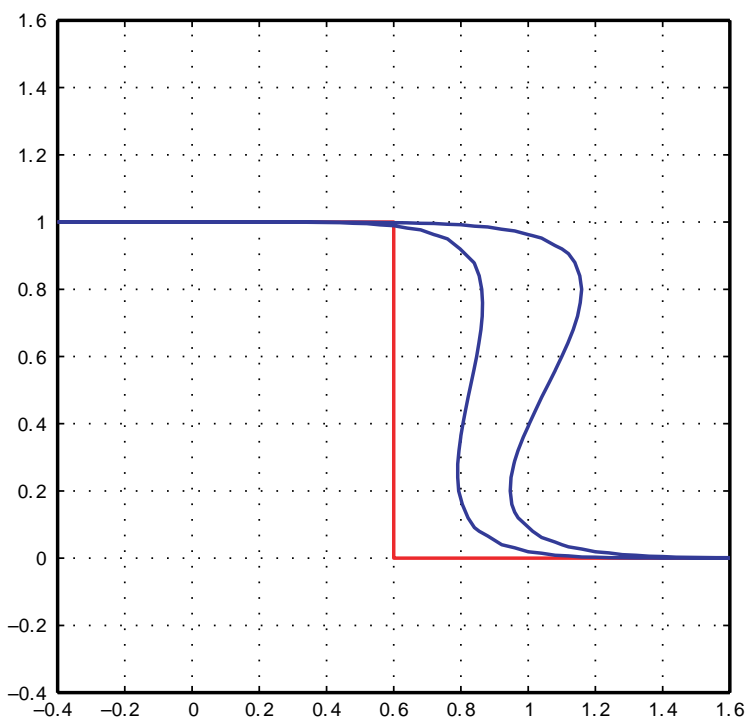

FiguRE 5. Incorrect (as expected) numerical solution to the Riemann problem for equation (2.2) with $u_{L}=1.0, u_{R}=0.0$, and $a=0.1$. We plotted the zero level set at times $t=0,0.5$, and 1.0.

Model equation $u_{t}+u u_{x}+a u\left|u_{x}\right|=0$. With $a \geq 1.0$, we know that this model equation retains the property that $\phi_{y} \geq 0$ for all time. Figure 4 show the computational result using (2.6) and third order WENO-LLF. The numerical solutions of this equation are computed with $a=2.0$. Finally, we show that our Lax-Friedrichs type scheme cannot be applied to compute solutions for equation with $a<1$. See Figure 5 .

2.3. Singular viscosity regularization. Consider the model equation (2.2) with $|a|<1$, and equation (2.3) with $v(y)$ nondecreasing. We know that it no longer has the minimum principle in $\phi_{y}$, and "overturning" or "folding" in its solution might develop.

Motivated by the work on a type of singular diffusion in [9, 10, 22, we will add a similar singular diffusion term in the $y$-direction to both our model equations:

$$
M|\nabla \phi| \frac{\partial}{\partial y}\left(\frac{\phi_{y}}{\left|\phi_{y}\right|}\right) \text {. }
$$

We first notice that this viscosity is activated only when $\operatorname{sign}\left(\phi_{y}\right)=\phi_{y} /\left|\phi_{y}\right|$ changes signs! With $M$ sufficiently large, this term $\partial\left(\operatorname{sign}\left(\phi_{y}\right)\right) / \partial y$ can be shown, at least formally, to concavify the primitive of the speed function on the vertical part of the level sets [12].

We briefly describe how to find the minimum value of $M$. Consider the primitive function $V(y)$ of the speed function $v(y)$ of equation (2.3) over $[a, b]$ that is a jump of $u$. Let $V^{*}$ be the function whose graph is the upper boundary of the convex hull of $V$. Let $V_{M}=V^{*}+2 M$. We claim that $M$ has to be large enough so that $V_{M}$ is tangent to or never crosses $V^{*}$. See Figure [6 for an example with $V(y)=y^{2} / 2$. Since the purpose of this paper is to provide the numerics, we refer the reader to the recent paper [12] of the second author for the formal reasoning. 


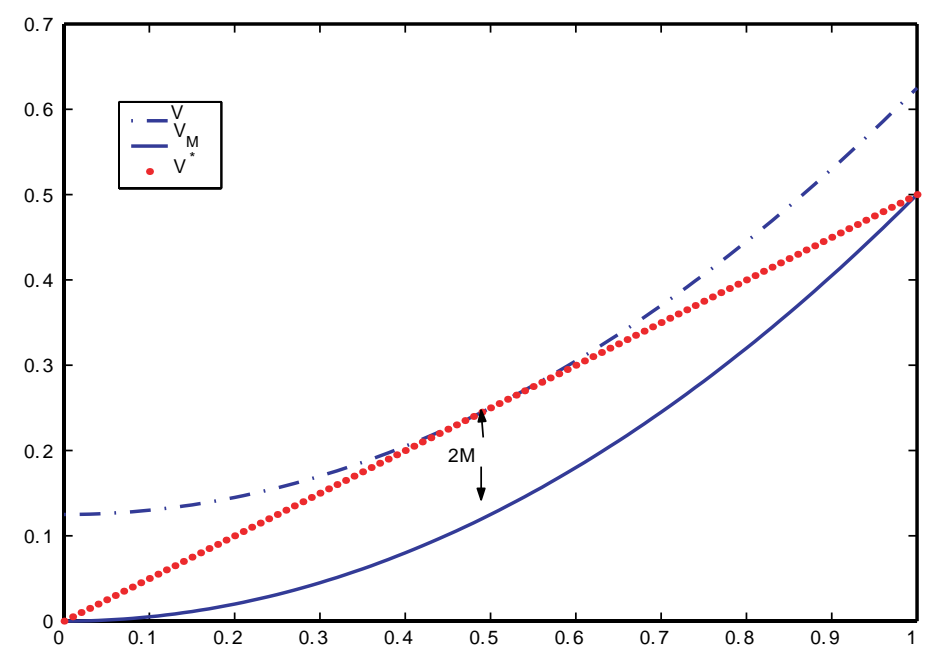

Figure 6. $V(y)=y^{2} / 2$ on $[0,1]$. The minimum value of $2 M$ should be $1 / 8$.

Alternatively, we describe another intuitive motivation behind this diffusion term. Consider the Heaviside function $y=H(x)$ and the level set function $\phi(x, y)$ for which this is the zero level set. If we treat the zero level set of $\phi$ locally as a function of $y$ wherever it is vertical, we see that the "overturning" will increase the total variation of $\{\phi=c\}$ as a function of $y$. This motivates the following regularization:

$$
\min _{\phi} \int\left|\phi_{y}\right| d y
$$

The corresponding Euler-Lagrange derivative is

$$
\frac{\partial}{\partial y}\left(\frac{\phi_{y}}{\left|\phi_{y}\right|}\right)
$$

To make the diffusion term geometrical, i.e., invariant of the choice of level set function, we multiply it by $|\nabla \phi|$ and arrive at the same diffusion term. Of course, in using this argument, we have to assume that the Hamiltonian is also the EulerLagrange derivative of some variational integral.

Now, let us go back to our model equation with this viscosity term:

$$
\phi_{t}-y \cdot\left(a \operatorname{sign}\left(\phi_{y}\right)\left|\phi_{x}\right|-\phi_{x}\right)=M|\nabla \phi| \frac{\partial}{\partial y}\left(\frac{\phi_{y}}{\left|\phi_{y}\right|}\right) .
$$

We use central differencing to approximate the singular diffusion term on the right hand side:

$$
\sqrt{\left(D_{x}^{0} \phi_{i, j}\right)^{2}+\left(D_{y}^{0} \phi_{i, j}\right)^{2}} \cdot \frac{\tanh \left(\gamma D_{y}^{+} \phi_{i, j}\right)-\tanh \left(\gamma D_{y}^{-} \phi_{i, j}\right)}{\Delta y},
$$

where the signum function $\phi_{y} /\left|\phi_{y}\right|$ is approximated by $\tanh \left(\gamma \phi_{y}\right)$ with $\gamma=1 / \Delta y$, and

$$
\tanh \left(\gamma D_{y}^{+} \phi_{i, j}\right)=\tanh \left(\gamma \frac{\phi_{i, j+1}-\phi_{i, j}}{\Delta y}\right)
$$


is an approximation of $\phi_{y} /\left|\phi_{y}\right|$ evaluated at $\left(x_{i}, y_{j+1 / 2}\right)$. Similarly $\tanh \left(\gamma D_{y}^{-} \phi_{i, j}\right)$ is an approximation for $\phi_{y} /\left|\phi_{y}\right|$ at $\left(x_{i}, y_{j-1 / 2}\right)$. The partial derivative $\phi_{x}$ on the left hand side is approximated by upwind differencing:

$|a|<1$ :

$$
|a| \geq 1:
$$

$$
\begin{array}{ll}
y \geq 0: & \phi_{x} \leftarrow D_{x}^{-} \phi, \\
y<0: & \phi_{x} \leftarrow D_{x}^{+} \phi,
\end{array}
$$

$$
\begin{aligned}
& \operatorname{sign}\left(D_{y}^{0} \phi\right) a y \leq 0: \phi_{x} \leftarrow\left(D_{-}^{x} \phi\right)^{+}-\left(D_{+}^{x} \phi\right)^{-}, \\
& \operatorname{sign}\left(D_{y}^{0} \phi\right) a y>0: \phi_{x} \leftarrow-\left(D_{-}^{x} \phi\right)^{-}+\left(D_{+}^{x} \phi\right)^{+} .
\end{aligned}
$$

Here, $p^{-}$denotes the negative part of $p$ (with sign) and $p^{+}$the positive part.

Because of the singular diffusion term, the stability condition becomes

$$
\frac{\Delta t}{\Delta x^{3}} \leq C_{M, H}
$$

where $C_{M, H}$ is a constant depending on the diffusion coefficient $M$ and the maximum values of $H_{\phi_{x}}$ and $H_{\phi_{y}}$.

Extension to higher order accuracy. Again, we may combine the central differencing approximation of the viscosity term and the WENO-LLF scheme described in the earlier section for numerical computation. This is needed for future generalization to more complex equations or to systems of equations, because upwinding is no longer easy.

2.3.1. Test on the model equation: $u_{t}+u u_{x}+a u\left|u_{x}\right|=0$. We first test our numerical scheme for the case $a=0.1$, which cannot be handled by the Lax-Friedrichs scheme (2.6). Figure 7 shows that the "overturning" is prevented, in contrast to the result shown in Figure 5 .

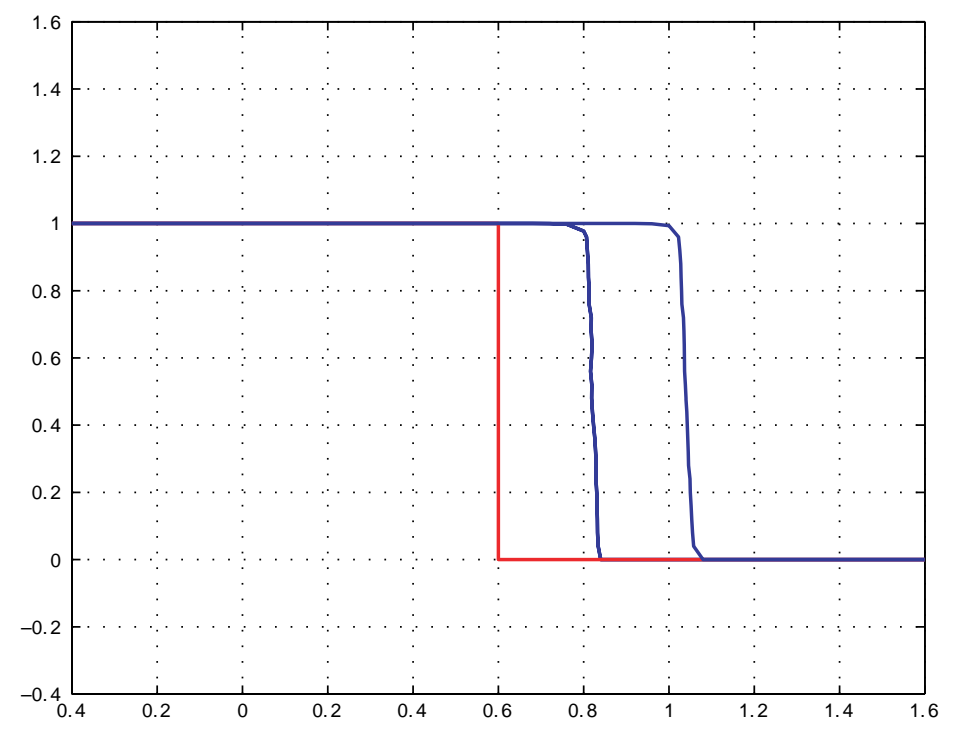

FiguRe 7. Numerical solution to the Riemann problem for equation (2.2) with $u_{L}=1.0, u_{R}=0.0, a=0.1$, and $M=0.2$. We plotted the zero level set at times $t=0,0.5$, and 1.0. 
2.3.2. Tests on conservation laws. As we have mentioned earlier, equation (2.1) with $a=0$ is equivalent to Burgers' equation in nonconservative form. Here we go one step further to demonstrate numerically that our regularization is equivalent to the entropy condition for conservation law equations.

We consider the conservation law

$$
u_{t}+f(u)_{x}=0
$$

with $f^{\prime} \geq 0$ and its corresponding linear level set equation

$$
\phi_{t}+f^{\prime}(y) \phi_{x}=0 \text {. }
$$

The numerical results shown in the following examples are obtained by plotting the zero contour of the numerical solution $\phi$ to the regularized equation:

$$
\phi_{t}+f^{\prime}(y) \phi_{x}=M|\nabla \phi| \frac{\partial}{\partial y}\left(\frac{\phi_{y}}{\left|\phi_{y}\right|}\right) .
$$

Burgers' equation. With $f(u)=u^{2} / 2$, we have the inviscid Burgers equation in nonconservative form. The corresponding level set equation becomes a linear transport equation with variable coefficient:

$$
\phi_{t}+y \phi_{x}=0
$$

It is then clear that the graph will overturn if $u$ is decreasing in $x$.

We consider the Riemann problem $u(x)=u_{L}=4.0$ for $x<0.0$ and $u(x)=$ $u_{R}=0.0$ for $x \geq 0.0$. See Figure 8 . The result shown in Figure 8 verifies the

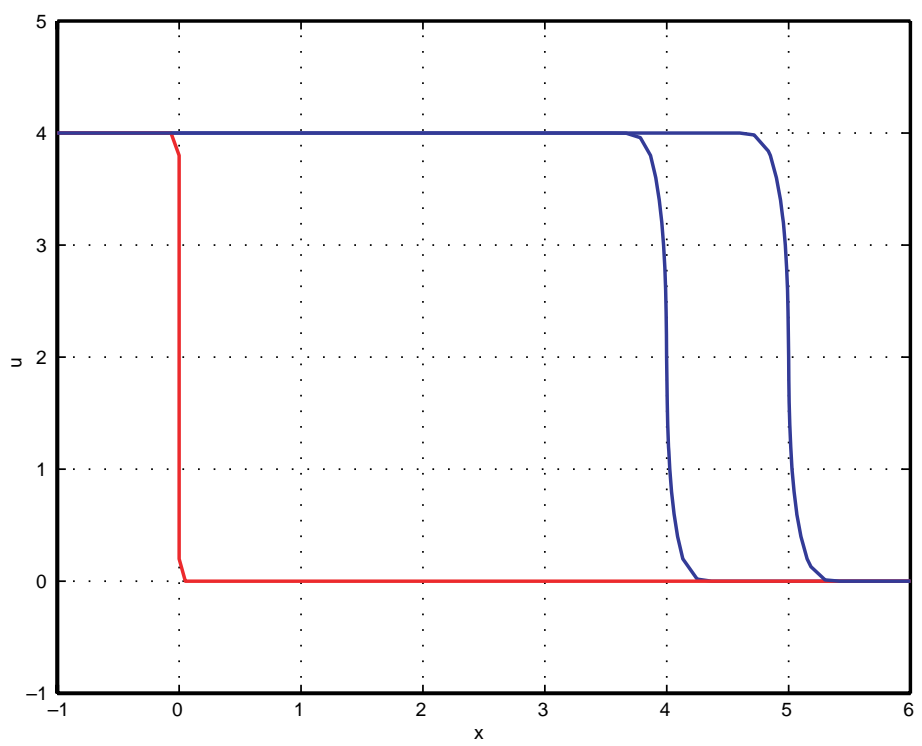

Figure 8. Numerical solution (WENO5-LLF) to the Riemann problem of Burgers' equation with $u_{L}=4.0, u_{R}=0.0$, and $M=2.1$. We plotted the zero level set at times $t=0,2.0$, and 2.5 . 

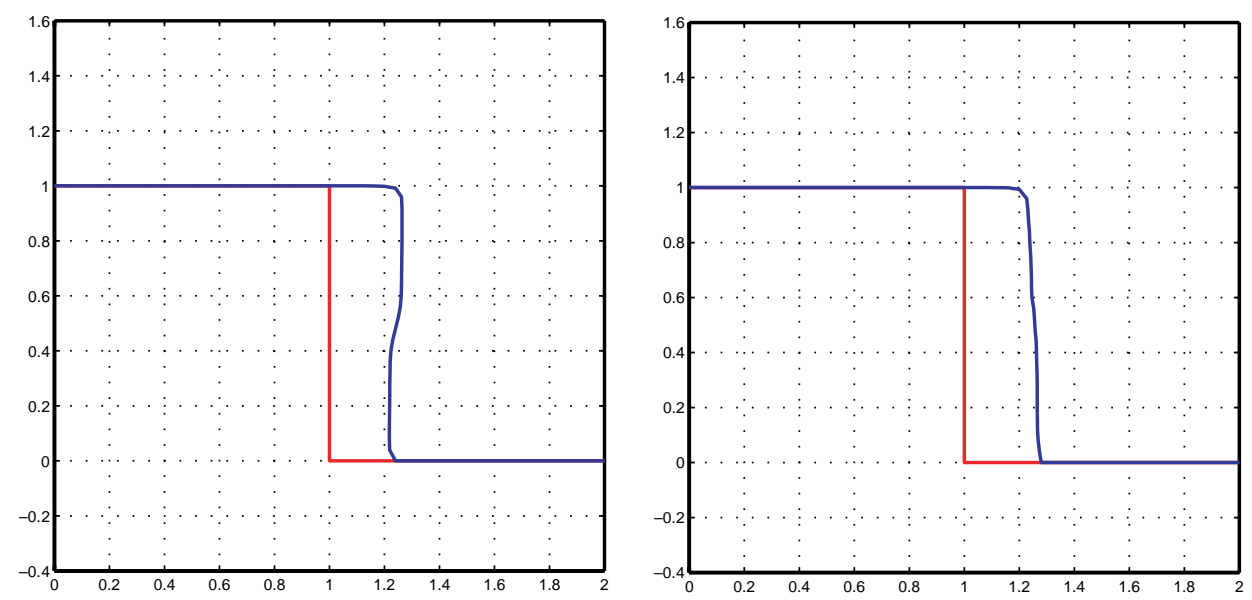

Figure 9. Numerical solution to the Riemann problem of Burgers' equation with $u_{L}=1.0, u_{R}=0.0$. We plotted the zero level sets at times $t=0$ and 0.5 obtained from $M=0.04$ and 1.0.

Rankine-Hugoniot shock speed:

$$
s=\frac{[f]}{[u]}=2.0 .
$$

Figure 9 shows a similar computation with $u_{L}=1.0, u_{R}=0.0$ and two different values of the diffusion coefficients $(M=0.04$ and $M=1.0)$. We can see that overturning will develop if $M$ is not large enough, and if it is sufficiently large, this coefficient does not affect the shock speed as predicted in [12] (the critical value for $M$ is 0.0625 in this case). We also compute the approximation obtained with no diffusion term (i.e., $M=0$ ) and plot it (green curve) against the one obtained from $M=0.2$ (blue curve), and show that the "equal-area" entropy condition is satisfied by the latter (blue curve). See Figure 10, Figure 11, shows the result of a Riemann problem with two shocks and a rarefaction. We also verify that the shocks in this case travel with the right speed.

Finally, we compute the solution to Burgers' equation starting with a sine curve: $\alpha \sin (\pi x)+\beta$. Figure [12 shows a first order approximation of the well-known $N$ wave starting with initial conditions using $\alpha=-0.8, \beta=0.0$. Our diffusion term successfully keeps the vertical part from overturning. In Figure 13, we obtained the numerical approximation, with $\alpha=-1.0$ and $\beta=0.5$, using the fifth order WENO local Lax-Friedrichs in space and third order TVD Runge-Kutta in time. One can see that the excessive drop in height caused by numerical diffusion using the standard first order Lax-Friedrichs method (Figure 12) is greatly reduced.

Consider the conservation law of equation 2.9 with initial condition $u_{0}(x)$. Until shock develops, the exact solution is defined implicitly by

$$
u=u_{0}\left(x-f^{\prime}(u) t\right) .
$$

Thus, for every fixed $(x, t)$, this can be thought of as a root-finding problem in $u$ using Newton's iterations

$$
u^{\nu+1}=u^{\nu}-\frac{f\left(u^{\nu}\right)}{f^{\prime}\left(u^{\nu}\right)},
$$




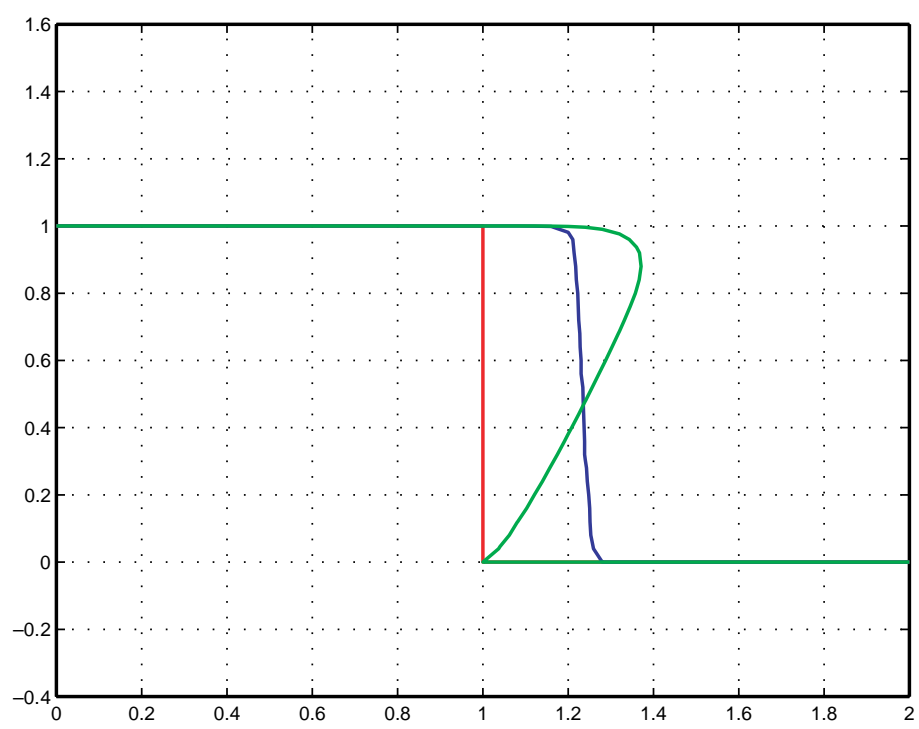

FiguRE 10. Numerical solution to the Riemann problem of Burgers' equation with $u_{L}=1.0, u_{R}=0.0$. We plotted the zero level sets at times $t=0$ and 0.5 obtained from $M=0.2$ and 0.0 .

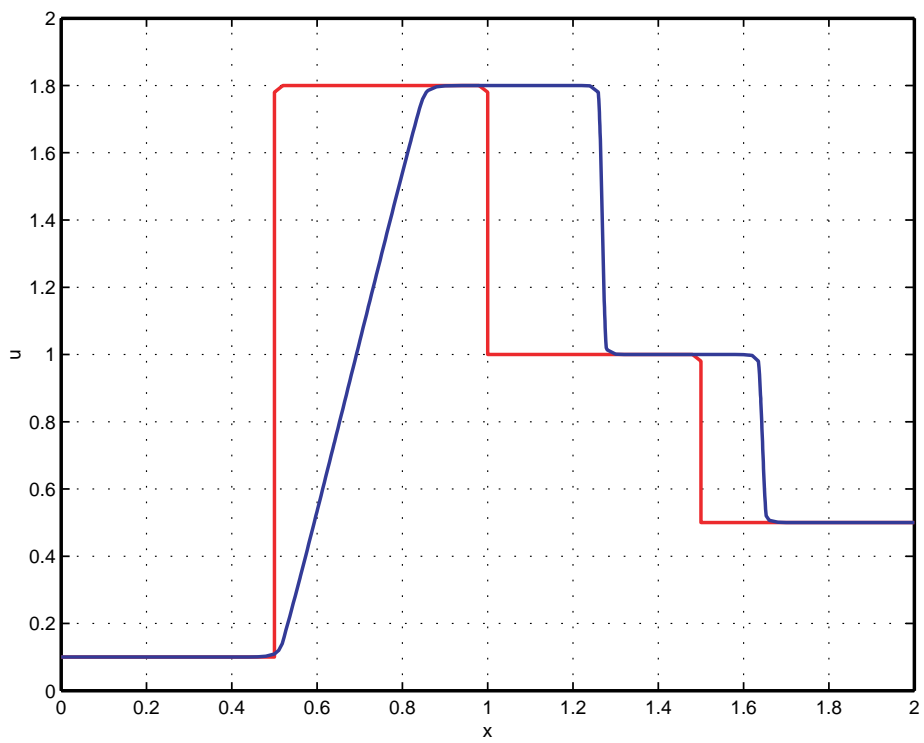

Figure 11. Numerical solution (WENO5-LLF) to the Riemann problem for Burgers' equation with $u_{L}=0.1, u_{M 1}=1.8, u_{M 2}=$ $1.0, u_{R}=0.5$, and $M=0.2$. We plotted the zero level set at times $t=0$ and 0.192 . 


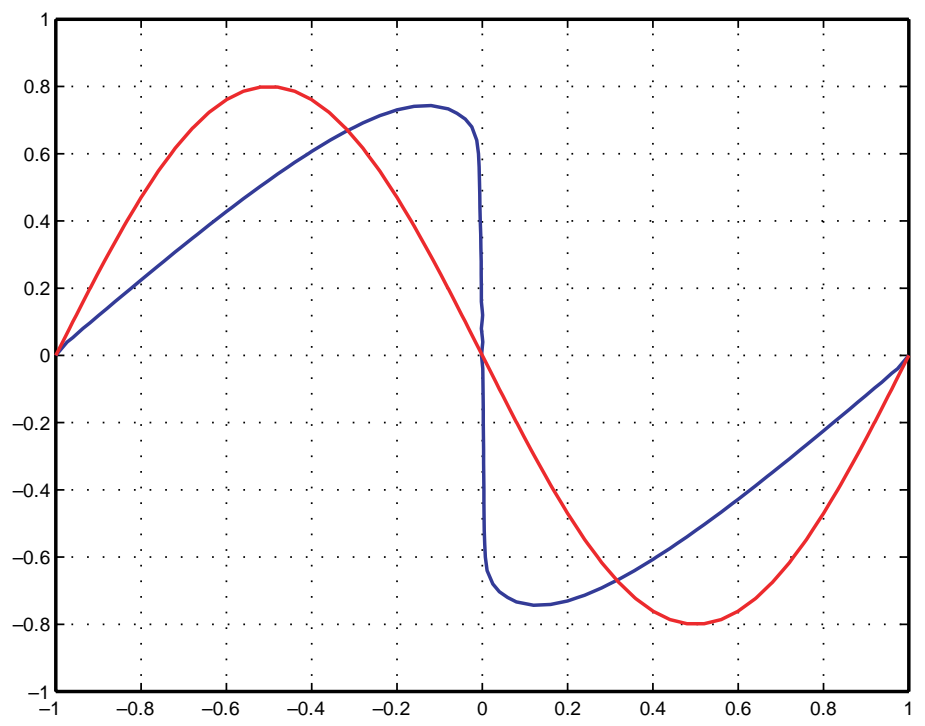

Figure 12. Numerical solution of Burgers' equation with sine wave as initial data. We plotted the zero level set at times $t=0$ and 0.5 .

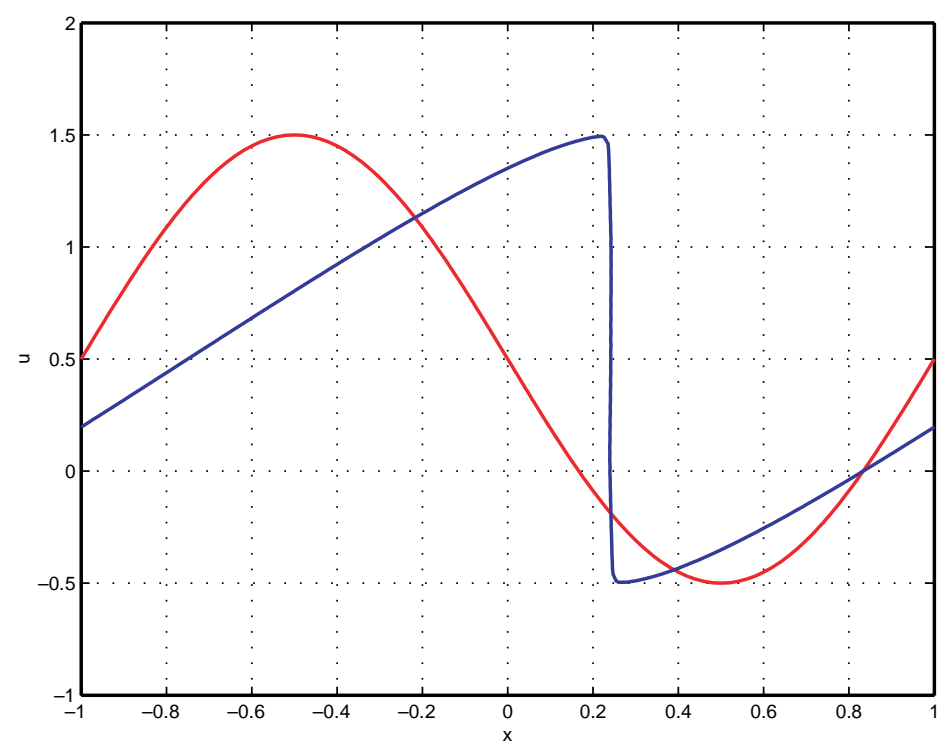

Figure 13. Numerical solution (WENO5-LLF) to the Burgers' equation with shifted sine wave as initial data. We plotted the zero level set at times $t=0$ and 0.5 .

where $f(u):=u_{0}\left(x-f^{\prime}(u) t\right)-u$. We use this simple iterative method to find the smooth solution to the machine accuracy and compare it to the solution obtained by our level approach. Some results are provided in Tables 1 and 2 , in which we 
TABLE 1. Numerical convergence (WENO5-LLF-RK3) of Burgers' equation with initial values $-0.8 \sin (\pi x)+0.5 . T=0.2$.

\begin{tabular}{|c|c|c|c|c|}
\hline & $d x=2 / 50$ & $d x=2 / 100$ & $d x=2 / 200$ & $d x=2 / 400$ \\
\hline max error & $5.99774 e-06$ & $3.65247 e-07$ & $3.39605 e-08$ & $3.88563 e-09$ \\
\hline rate & & 4.0375 & 3.4269 & 3.1276 \\
\hline
\end{tabular}

TABLE 2. Numerical convergence of WENO5-LLF-RK3 on Burgers' equation with initial values $-0.8 \sin (\pi x), M=0.2, T=0.384$.

\begin{tabular}{|c|c|c|c|}
\hline & $d x=2 / 25$ & $d x=2 / 50$ & $d x=2 / 100$ \\
\hline max error & 0.007603086 & 0.0009753479 & $9.64295 e-05$ \\
\hline rate & & 2.9626 & 3.3384 \\
\hline
\end{tabular}

use a third order linear interpolation to approximate the location of the zero level curve on each grid point. In particular, Table 2 shows a third order convergence of the numerical solutions in the region excluding a $5 \Delta x$ neighborhood of the shock.

Buckley-Leverett equation. Finally, we test our numerical method for equation (2.11) to substantiate our assertion that the singular diffusion term minimally concavifies the flux function $f$ over the jump interval. We solve the Riemann problem of the conservation law

$$
u_{t}+f(u)_{x}=0
$$

with

$$
f(u)=\frac{u^{2}}{u^{2}+a(1-u)^{2}}, \quad a>0, u \in[0,1] .
$$

and $u_{L}=1.0, u_{R}=0.0$.

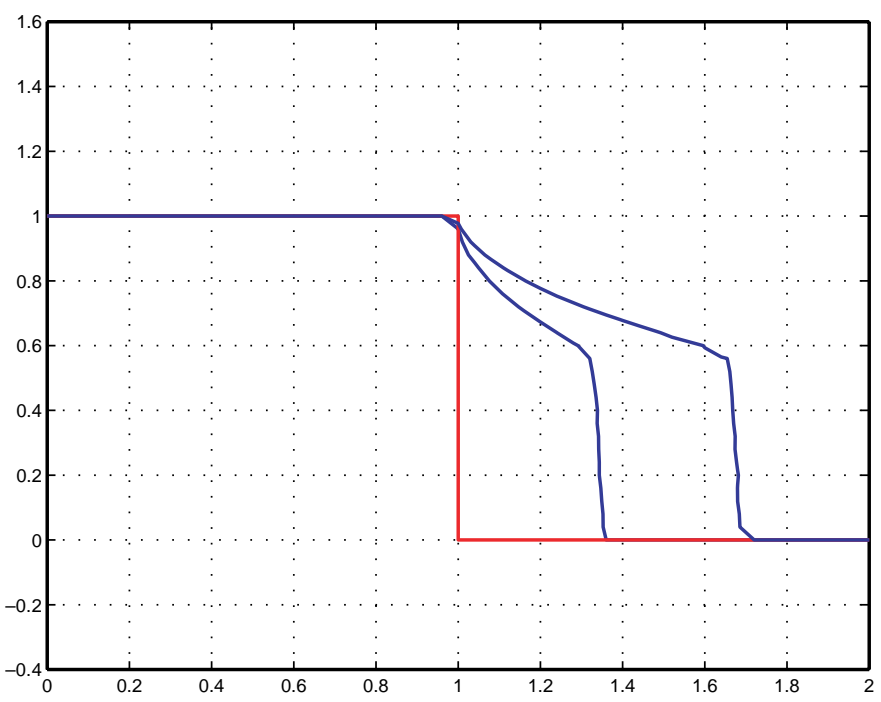

Figure 14. Numerical solution to the Riemann problem for the Buckley-Leverett equation with $u_{L}=1.0, u_{R}=0.0$ and $a=0.5$. We plotted the zero level set at times $t=0,0.25$, and 0.5 . 


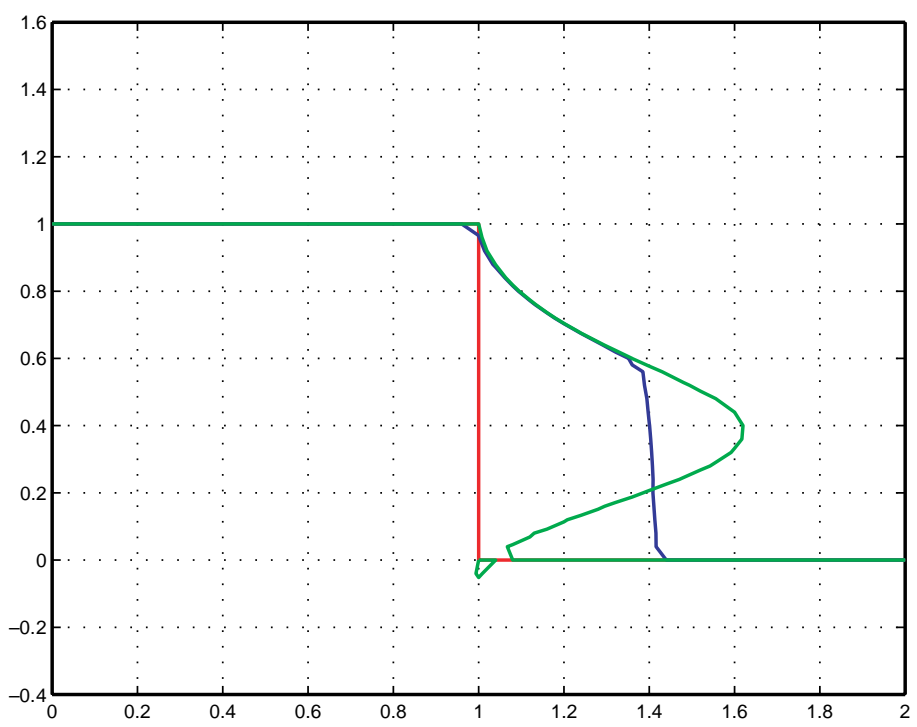

FiguRe 15. Numerical solution to the Riemann problem for the Buckley-Leverett equation with $u_{L}=1.0, u_{R}=0.0$ and $a=0.5$. We plotted the zero level sets at times $t=0$ and 0.3 obtained from $M=0.2$ and 0.0. The little fragment of contour at the lower part of the jump is due to the contour plotter.

The upper boundary of the convex hull of $\operatorname{sg}(f)$ consists of a straight line segment $L$ from $(0,0)$ to $\left(u^{*}, f\left(u^{*}\right)\right)$ followed by $(u, f(u))$ for $u \in\left[u^{*}, 1\right]$, where $L$ is a tangent line of $f(u)$. See Figure 2, The slope of $L$ is also the correct shock speed for the Riemann problem. With $a=0.5$, a simple calculation shows that $u^{*}=1 / \sqrt{3} \doteq$ 0.57735 .

Figure 14 shows the expected rarefaction from $u_{L}$ to $u^{*}$ and a shock between $u^{*}$ and $u_{R}$. Figure 15 shows an overlap of the solutions obtained with and without regularization. One can observe that the "equal-area" entropy condition is satisfied.

2.3.3. A two-dimensional example. To show that our numerical schemes extend naturally to higher dimensions, we show our numerical solutions for the equation

$$
u_{t}-u \sqrt{1+u_{x}^{2}+u_{y}^{2}}=0 .
$$

When $u$ is continuous, this is motion in the normal direction of the graph of $u$ with speed $u$. The corresponding level set equation

$$
\phi_{t}+z \sqrt{\phi_{x}^{2}+\phi_{y}^{2}+\phi_{z}^{2}}=0
$$

gives a straightforward geometrical interpretation of the solution as level sets of $\phi$. Results are shown in Figures [16] and [7] In both figures, the initial data are represented by the blue surfaces (one cubical and the other spherical) and the solutions during later times are represented by the green and yellow surfaces. The left subfigures are computed without the regularization, whereas the right ones are regularized by the singular diffusive term $M|\nabla \phi| \partial / \partial\left(\phi_{z} /\left|\phi_{z}\right|\right)$. 

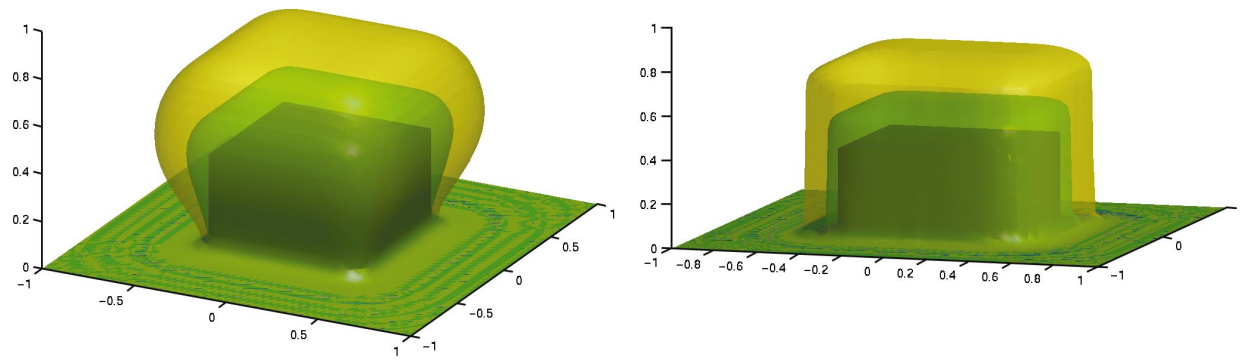

FiguRE 16.
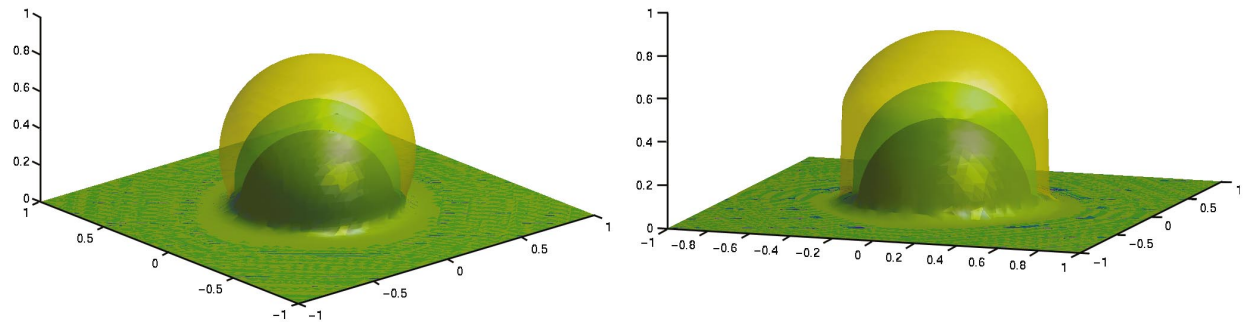

FigURE 17.

2.3.4. The vanishing viscosity approach. Consider the Lax-Friedrichs type scheme of the following form:

$$
u_{i}^{n+1}=u_{i}^{n}-\Delta t H\left(x_{i}, u_{i}^{n}, D_{x}^{0} u_{i}^{n}\right)+c \Delta_{x}^{+} \Delta_{x}^{-} u_{i}^{n} / 2,
$$

where $\Delta_{x}^{ \pm} u_{i}^{n}$ denotes the undivided forward/backward difference of $u_{i}^{n}$ and $0 \leq c \leq$ 1. With

$$
\Delta t \leq \min \left(\frac{1-c}{\left\|H_{u}\right\|_{\infty}}, \frac{c \Delta x}{\left\|H_{u_{x}}\right\|_{\infty}}\right)
$$

the scheme is monotone and seems to yield convergent approximations for the equations with $H_{u} \geq 0$.

However, this scheme is not suitable for the HJ equations, whose solutions develop shocks. Figure 18 shows the numerical approximations using (2.12) with different values of $c$ and fairly small grid size. The leftmost curve is the initial data. The remaining curves from left to right are obtained using $c=0.1,0.99$, and 0.9 , respectively. One can see that the numerical solutions converge to different functions.

We maintain that our level set approach is no less efficient since we can do the computation locally around the zero level curve 28. Also, the level set approach is more "natural" since it is a part of the theoretical notion of solutions to the HJ equations that we are concerned with. 


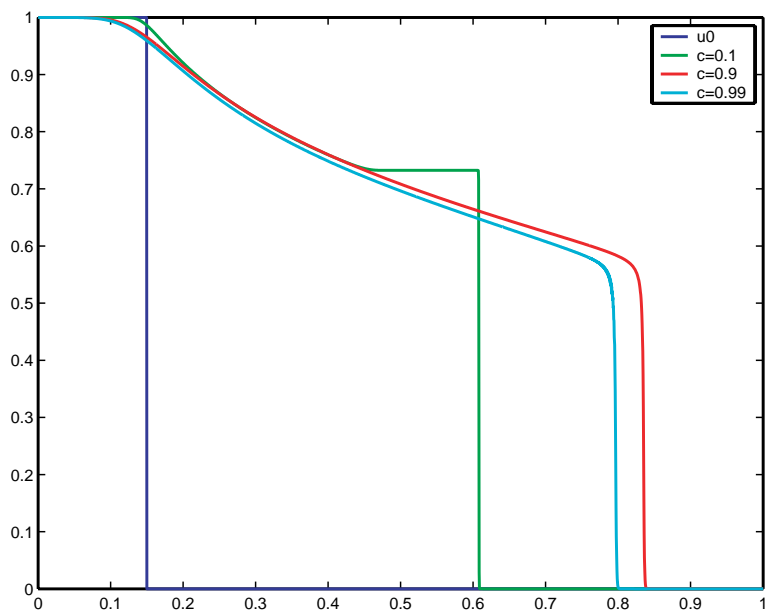

FiguRE 18. The numerical solutions of the Buckley-Leverett equation in nonconservative form obtained from the monotone LaxFriedrichs scheme (2.12). The approximations are computed to $t=0.1$ on $[0,1]$ with 2,500 grid points.

\section{Summary}

In this paper, we provided two classes of finite difference methods for the computation of the semicontinuous L-solution of a class of HJ equations. By studying the level set equation derived from the HJ equations, we pointed out the necessary condition for the validity of the solution defined as the zero contour line of the level set function. We have also discussed the geometrical interpretation of the motion of the solution embedded in the level set function. The remarks provide hints on how to regularize the zero level curve motion so that it can be interpreted as the graph of a function.

For the class of HJ equations with $H_{y} \geq 0$, we applied a straightforward LaxFriedrichs type scheme with possibility of extension to higher order accuracy. We showed numerically that the singular diffusion term $|\nabla \phi| \partial\left(\phi_{y} /\left|\phi_{y}\right|\right) / \partial y$ can be applied to compute the shock solution for the class of HJ equations we considered. In particular, we numerically verified that our numerical schemes yield approximations compatible with the entropy solution of a conservation law equation with nonconvex flux. Of course, we have also shown the extension of our numerical schemes to higher order WENO-local Lax-Friedrichs schemes.

Finally, we remark here that our numerical schemes for the derived level set equations can be computed locally around the zero level curve using the technique described in [28] for efficiency.

\section{Systems of CONSERVATION LAWS}

We generalize the result of our singular viscosity to study the solution of conservation law systems and the link to Riemann invariants. Here we briefly describe how we approach this problem. 
Let $\vec{u}=(u, v) \in \mathbb{R}^{2}, \phi(t, x, y): \mathbb{R}^{+} \times \mathbb{R} \times \mathbb{R}^{2} \mapsto \mathbb{R}^{2}$ be the vector-valued level set function such that $\phi(t, x, \vec{u}(t, x))=0$. The system

$$
\vec{u}_{t}+A(\vec{u}) \vec{u}_{x}=0
$$

can be formally translated to

$$
\phi_{t}+\phi_{y} A(y) \phi_{y}^{-1} \phi_{x}=0 .
$$

We shall use the Riemann invariants for the $2 \times 2$ system to diagonalize $A(y)$ and desingularize the term $\phi_{y}^{-1}$.

We propose a singular diffusion term similar to the scalar one we used. With an abuse of notation, this term can be written as

$$
\left|\nabla_{x, y} \phi\right| \nabla_{y} \cdot\left(\left|\nabla_{y} \phi\right|^{-1} \nabla_{y} \phi\right),
$$

where $\nabla_{x, y} \phi$ is the Jacobian matrix of $\phi$ with respect to $x$ and $y, \nabla_{y} \phi=\phi_{y}$ is the Jacobian matrix of $\phi$, and $|A|:=\sqrt{A A^{*}}$ is the Euclidean norm of the matrix $A$.

Acknowledgments. The authors YT and SO would like to thank Paul Burchard for very useful conversations on this topic, and Chi-Wang Shu for his careful reading of the first draft.

\section{APPENDIX: INITIALIZING THE LEVEL SET FUNCTION}

Since the introduction of level set methods [26, several techniques have been developed to compute the level set function for a given curve $\Gamma$ (this is called the initialization step in standard level set jargon). We point out several such techniques for completeness of the numerics.

If the curve $\Gamma$ in question is the graph of a smooth function $u(x)$, the easiest way to initialize the level set function $\phi(x, y)$ is simply

$$
\phi(x, y)=y-u(x) .
$$

However, if $u(x)$ is only piecewise smooth, we cannot use the same approach to initialize $\phi$, since the function obtained this way becomes discontinuous.

The next easy way is to use the signed distance function of the upper boundary of $\operatorname{sg}(u)$ as the level set function; that is, consider the upper boundary of $\operatorname{sg}(u)$ as a continuous curve $\Gamma \in \mathbb{R}^{2}$, and set

$$
\phi(x, y)=m(x, y) \operatorname{dist}((x, y), \Gamma),
$$

where $m(x, y)=\operatorname{sgn}(y-u(x))$. We notice that the level set function constructed this way is Lipschitz continuous everywhere.

There are a variety of techniques to compute the distance function. For example, the fast marching method [17, [29], [33] can be used to find $\phi$ quickly by constructing a first order approximation of the solution of the eikonal equation

$$
|\nabla \phi|=1 .
$$

For a piecewise linear curve, the method described in [32] can be used for accurate and rapid construction of the distance function. The initialization of the numerical examples presented in this paper are all constructed this way. 


\section{REFERENCES}

1. Martino Bardi and Italo Capuzzo-Dolcetta, Optimal control and viscosity solutions of Hamilton-Jacobi-Bellman equations, Birkhäuser Boston Inc., Boston, MA, 1997, With appendices by Maurizio Falcone and Pierpaolo Soravia. MR 99e:14001

2. Guy Barles, Discontinuous viscosity solutions of first-order Hamilton-Jacobi equations: a guided visit, Nonlinear Anal. 20 (1993), no. 9, 1123-1134. MR 94d:49047

3. Guy Barles, Solution de viscosité des équations de Hamilton-Jacobi, Springer-Verlag, 1994. MR 2000b:49054

4. E. N. Barron and R. Jensen, Semicontinuous viscosity solutions for Hamilton-Jacobi equations with convex Hamiltonians, Comm. Partial Differential Equations 15 (1990), no. 12, 1713-1742. MR 91b:35069

5. C Caratheodory, Calculus of varieties of partial differential equations of the first order, Chelsea, 1982. MR 33:597, MR 38:590 (earlier ed.)

6. M.G. Crandall and P.L. Lions, Two approximations of solutions of Hamilton-Jacobi equations, Mathematics of Computation 43 (1984), 1-19. MR 86j:65121

7. Michael G. Crandall and Pierre-Louis Lions, Viscosity solutions of Hamilton-Jacobi equations, Trans. Amer. Math. Soc. 277 (1983), no. 1, 1-42. MR 85g:35029

8. Lawrence C. Evans, A geometric interpretation of the heat equation with multivalued initial data, SIAM J. Math. Anal. 27 (1996), no. 4, 932-958. MR 98g:35092

9. Mi-Ho Giga and Yoshikazu Giga, Crystalline and level set flow-convergence of a crystalline algorithm for a general anisotropic curvature flow in the plane, Free boundary problems: theory and applications, I (Chiba, 1999), Gakkōtosho, Tokyo, 2000, pp. 64-79. MR 2002f:53117

10. Mi-Ho Giga, Yoshikazu Giga, and Ryo Kobayashi, Very singular diffusion equations, Advanced Studies in Pure Mathematics 31, 2001, pp. 93-125.

11. Y. Giga, S. Goto, H. Ishii, and M.-H. Sato, Comparison principle and convexity preserving properties for singular degenerate parabolic equations on unbounded domains, Indiana Univ. Math. J. 40 (1991), no. 2, 443-470. MR 92h:35010

12. Yoshikazu Giga, Shocks and very strong vertical diffusion, To appear, Proc. of international conference on partial differential equations in celebration of the seventy fifth birthday of Professor Louis Nirenberg, Taiwan, 2001.

13. _ Viscosity solutions with shocks, Hokkaido Univ. Preprint Series in Math. (2001), no. 519.

14. Yoshikazu Giga and Moto-Hiko Sato, A level set approach to semicontinuous viscosity solutions for Cauchy problems, Comm. Partial Differential Equations 26 (2001), no. 5-6, 813-839.

15. Ami Harten, High resolution schemes for hyperbolic conservation laws, J. Comput. Phys. 49 (1983), no. 3, 357-393. MR 84g:65115

16. Ami Harten, Björn Engquist, Stanley Osher, and Sukumar R. Chakravarthy, Uniformly highorder accurate essentially nonoscillatory schemes. III, J. Comput. Phys. 71 (1987), no. 2, 231-303. MR 90a:65199

17. J. Helmsen, E. Puckett, P. Colella, and M. Dorr, Two new methods for simulating photolithography development in 3d, SPIE 2726, 1996, pp. 253-261.

18. Hitoshi Ishii, Hamilton-Jacobi equations with discontinuous Hamiltonians on arbitrary open sets, Bull. Fac. Sci. Engrg. Chuo Univ. 28 (1985), 33-77. MR 87k:35055

19. - Existence and uniqueness of solutions of Hamilton-Jacobi equations, Funkcial. Ekvac. 29 (1986), no. 2, 167-188. MR 88c:35037

20. _ Perron's method for Hamilton-Jacobi equations, Duke Math. J. 55 (1987), no. 2, 369-384. MR 89a:35053

21. Guang-Shan Jiang and Danping Peng, Weighted ENO schemes for Hamilton-Jacobi equations, SIAM J. Sci. Comput. 21 (2000), no. 6, 2126-2143 (electronic). MR 2001e:65124]

22. R. Kobayashi and Y. Giga, Equations with singular diffusivity, J. Statist. Phys. 95 (1999), no. 5-6, 1187-1220. MR 2001f:82077

23. Peter D. Lax, Hyperbolic systems of conservation laws and the mathematical theory of shock waves, Society for Industrial and Applied Mathematics, Philadelphia, Pa., 1973, Conference Board of the Mathematical Sciences Regional Conference Series in Applied Mathematics, No. 11. MR 50:2709]

24. Chi-Tien Lin and Eitan Tadmor, High-resolution nonoscillatory central schemes for Hamilton-Jacobi equations, SIAM J. Sci. Comput. 21 (2000), no. 6, 2163-2186 (electronic). MR 2001e:65125 
25. Stanley Osher, A level set formulation for the solution of the Dirichlet problem for HamiltonJacobi equations, SIAM J Math Anal 24 (1993), no. 5, 1145-1152. MR 94h:35039

26. Stanley Osher and James A. Sethian, Fronts propagating with curvature-dependent speed: algorithms based on Hamilton-Jacobi formulations, J. Comput. Phys. 79 (1988), no. 1, 12-49. MR 89h:80012

27. Stanley Osher and Chi-Wang Shu, High-order essentially nonoscillatory schemes for Hamilton-Jacobi equations, SIAM J. Numer. Anal. 28 (1991), no. 4, 907-922. MR 92e:65118

28. Danping Peng, Barry Merriman, Stanley Osher, Hongkai Zhao, and Myungjoo Kang, A PDE-based fast local level set method, J. Comput. Phys. 155 (1999), no. 2, 410-438. MR 2000j:65104

29. J.A. Sethian, Fast marching level set methods for three dimensional photolithography development, SPIE 2726, 1996, pp. 261-272.

30. Chi-Wang Shu and Stanley Osher, Efficient implementation of essentially nonoscillatory shock-capturing schemes. II, J. Comput. Phys. 83 (1989), no. 1, 32-78. MR 90i:65167

31. Panagiotis E. Souganidis, Approximation schemes for viscosity solutions of Hamilton-Jacobi equations, J. Differential Equations 59 (1985), no. 1, 1-43. MR 86k:35028

32. Yen-Hsi Richard Tsai, Rapid and accurate computation of the distance function using grids, UCLA CAM Report 00 (2000), no. 36.

33. John Tsitsiklis, Efficient algorithms for globally optimal trajectories, IEEE Transactions on Automatic Control 40 (1995), no. 9, 1528-1538. MR 96d:49039

34. Bram van Leer, Towards the ultimate conservative difference schemes V, J. Comput. Phys. 32 (1979), 102-136. MR 90h:70003

Department of Mathematics, University of California los Angeles, Los Angeles, CALIFORNia 90095

E-mail address: ytsai@math.ucla.edu

Department of Mathematics, Hokkaido University, Sapporo 060-0810, Japan

E-mail address: giga@math.sci.hokudai.ac.jp

Department of Mathematics, University of California los Angeles, Los Angeles, CALIFORNIA 90095

E-mail address: sjo@math.ucla.edu 